\title{
Inversion of Weinstein intertwining operator and its dual using Weinstein wavelets
}

\author{
Abdessalem Gasmi \& Hassen Ben Mohamed \& Néji Bettaibi
}

\begin{abstract}
In this paper, we consider the Weinstein intertwining operator $\mathcal{R}_{W}^{\alpha, d}$ and its dual ${ }^{t} \mathcal{R}_{W}^{\alpha, d}$. Using these operators, we give relations between the Weinstein and the classical continuous wavelet transforms. Finally, using the Weinstein continuous wavelet transform, we deduce the formulas which give the inverse operators of $\mathcal{R}_{W}^{\alpha, d}$ and ${ }^{t} \mathcal{R}_{W}^{\alpha, d}$.
\end{abstract}

\section{INTRODUCTION}

In this paper, we consider the Weinstein operator $\Delta_{W}^{\alpha, d}$ defined on $\mathbb{R}_{+}^{d+1}=$ $\left.\mathbb{R}^{d} \times\right] 0,+\infty[$, by:

$$
\Delta_{W}^{\alpha, d}=\sum_{i=1}^{d+1} \frac{\partial^{2}}{\partial x_{i}^{2}}+\frac{2 \alpha+1}{x_{d+1}} \frac{\partial}{\partial x_{d+1}}=\Delta_{d}+L_{\alpha}, \alpha>-\frac{1}{2},
$$

where $\Delta_{d}$ is the Laplacian for the $d$ first variables and $L_{\alpha}$ is the Bessel operator for the last variable defined on $] 0,+\infty[$ by :

$$
L_{\alpha} u=\frac{\partial^{2} u}{\partial x_{d+1}^{2}}+\frac{2 \alpha+1}{x_{d+1}} \frac{\partial u}{\partial x_{d+1}}=\frac{1}{x_{d+1}^{2 \alpha+1}} \frac{\partial}{\partial x_{d+1}}\left[x_{d+1}^{2 \alpha+1} \frac{\partial u}{\partial x_{d+1}}\right] .
$$

The Weinstein operator $\Delta_{W}^{\alpha, d}$, mostly referred to as the Laplace-Bessel differential operator is now known as an important operator in analysis, due its

Key Words: Weinstein, intertwining operator, continuous wavelet transform.

2010 Mathematics Subject Classification: Primary 42B10, 42B35; Secondary $42 B 35$.

Received: 4.08 .2014

Accepted: 20.10 .2014 . 
applications in pure and applied Mathematics, especially in Fluid Mechanics [7].

The relevant harmonic analysis associated with the Bessel differential operator $L_{\alpha}$ goes back to S. Bochner, J. Delsarte, B. M. Levitan and has been studied by many other authors such as J. Löfström and J. peetre [11], I. Kipriyanov [9], K. Stempak [14], K. Trimèche [15], I. A. Aliev and B. Rubin [1].

The Weinstein intertwining operator is the operator $\mathcal{R}_{W}^{\alpha, d}$ defined on $\mathcal{E}_{*}\left(\mathbb{R}^{d+1}\right.$ ) (the space of $C^{\infty}$-functions on $\mathbb{R}^{d+1}$, even with respect to the last variable) by

$$
\forall x \in \mathbb{R}_{+}^{d+1}, \mathcal{R}_{W}^{\alpha, d}(f)(x)=a_{\alpha} \int_{0}^{1}\left(1-t^{2}\right)^{\alpha-\frac{1}{2}} f\left(x^{\prime}, t x_{d+1}\right) d t,
$$

where $a_{\alpha}$ is the constant given by :

$$
a_{\alpha}=\frac{2 \Gamma(\alpha+1)}{\sqrt{\pi} \Gamma\left(\alpha+\frac{1}{2}\right)} .
$$

The dual of the operator $\mathcal{R}_{W}^{\alpha, d}$ is defined on $\mathcal{D}_{*}\left(\mathbb{R}^{d+1}\right.$ ) ( the space of $C^{\infty}$ functions on $\mathbb{R}^{d+1}$ which are of compact support, even with respect to the last variable $)$ is given for all $y=\left(y^{\prime}, y_{d+1}\right) \in \mathbb{R}_{+}^{d+1}$ by :

$$
{ }^{t} \mathcal{R}_{W}^{\alpha, d}(f)(y)=a_{\alpha} \int_{y_{d+1}}^{+\infty}\left(s^{2}-y_{d+1}^{2}\right)^{\alpha-\frac{1}{2}} f\left(y^{\prime}, s\right) s d s .
$$

$\mathcal{R}_{W}^{\alpha, d}$ and ${ }^{t} \mathcal{R}_{W}^{\alpha, d}$ are topological ismorphism on $\mathcal{E}_{*}\left(\mathbb{R}^{d+1}\right)$ and $\mathcal{D}_{*}\left(\mathbb{R}^{d+1}\right)$, respectively, and they serve as transmutation operators between the Weinstein operator and the Laplacian operator on $\mathbb{R}^{d+1}$.

The main objective of this paper is to establish the inverse operators of the operators $\mathcal{R}_{W}^{\alpha, d}$ and ${ }^{t} \mathcal{R}_{W}^{\alpha, d}$ in new functional spaces, using some pseudodifferential operators and using Weinstein wavelets.

For this purpose, we define and study the Weinstein continuous wavelet transform $S_{g}^{W}$ and we establish for this transform a Plancherel and an inversion formulas. Using the operator $\mathcal{R}_{W}^{\alpha, d}$ and its dual ${ }^{t} \mathcal{R}_{W}^{\alpha, d}$, we give relations between $S_{g}^{W}$ and the classical continuous wavelet transform $S_{g}$. Using the inversion formulas of the transforms $S_{g}^{W}$ and $S_{g}$, we deduce the formulas which give the inverse operators of $\mathcal{R}_{W}^{\alpha, d}$ and ${ }^{t} \mathcal{R}_{W}^{\alpha, d}$, using wavelets.

The contents of this paper is as follows :

In the second Section, we recall some basic harmonic analysis results related with the Weinstein operator developed in [2], [3] and [4].

In the third Section, we list some basic properties of the Weinstein intertwining operator $\mathcal{R}_{W}^{\alpha, d}$ and its dual ${ }^{t} \mathcal{R}_{W}^{\alpha, d}$. 
In the fourth Section, we define and characterize new spaces of functions $\mathcal{S}_{*, 0}\left(\mathbb{R}^{d+1}\right)$ and $\mathcal{S}_{*, 0}^{0}\left(\mathbb{R}^{d+1}\right)$ on which the operator $\mathcal{R}_{W}^{\alpha, d}$ and its dual ${ }^{t} \mathcal{R}_{W}^{\alpha, d}$ are bijective.

In the fifth Section, we give the definition of the Weinstein continuous wavelet transform $S_{g}^{W}$ and we establish a Plancherel and an inversion formulas. Using the operator $\mathcal{R}_{W}^{\alpha, d}$ and its dual ${ }^{t} \mathcal{R}_{W}^{\alpha, d}$, we give relations between $S_{g}^{W}$ and the classical continuous wavelet transform $S_{g}$. Finally, in the last Section, using the inversion formulas of the transforms $S_{g}^{W}$ and $S_{g}$, we deduce the formulas which give the inverse operators of $\mathcal{R}_{W}^{\alpha, d}$ and ${ }^{t} \mathcal{R}_{W}^{\alpha, d}$.

\section{Harmonic analysis associated with the Weinstein-Laplace operator}

In this section, we shall collect some results and definitions from the theory of the harmonic analysis associated with the Weinstein operator $\Delta_{W}^{\alpha, d}$ defined on $\mathbb{R}_{+}^{d+1}$ by the relation (1). Main references are $([2,3,4,5,8,12,13])$.

Let us begin by the following result, which gives the eigenfunction $\Psi_{\lambda}^{\alpha, d}$ of the Weinstein operator $\Delta_{W}^{\alpha, d}$.

Proposition 1.

For all $\lambda=\left(\lambda_{1}, \lambda_{2}, \ldots, \lambda_{d+1}\right) \in \mathbb{R}_{+}^{d+1}$, the system

$$
\left\{\begin{array}{c}
\frac{\partial^{2} u}{\partial x_{j}^{2}}(x)=-\lambda_{j}^{2} u(x), \text { if } 1 \leq j \leq d \\
L_{\alpha} u(x)=-\lambda_{d+1}^{2} u(x), \\
u(0)=1, \frac{\partial u}{\partial x_{d+1}}(0)=0 \text { and } \frac{\partial u}{\partial x_{j}}(0)=-i \lambda_{j}, \text { if } 1 \leq j \leq d
\end{array}\right.
$$

has a unique solution $\Psi_{\lambda}^{\alpha, d}$ given by

$$
\forall z \in \mathbb{C}^{d+1}, \Psi_{\lambda}^{\alpha, d}(z)=e^{-i\left\langle z^{\prime}, \lambda^{\prime}\right\rangle} j_{\alpha}\left(\lambda_{d+1} z_{d+1}\right),
$$

where $z=\left(z^{\prime}, x_{d+1}\right), z^{\prime}=\left(z_{1}, z_{2}, \ldots, z_{d}\right)$ and $j_{\alpha}$ is the normalized Bessel function of index $\alpha$, defined by :

$$
\forall \xi \in \mathbb{C}, j_{\alpha}(\xi)=\Gamma(\alpha+1) \sum_{n=0}^{\infty} \frac{(-1)^{n}}{n ! \Gamma(n+\alpha+1)}\left(\frac{\xi}{2}\right)^{2 n} .
$$

Remark 1. The Weinstein kernel $\Lambda_{\alpha, d}:(\lambda, z) \mapsto \Psi_{\lambda}^{\alpha, d}(z)$ has a unique extention to $\mathbb{C}^{d+1} \times \mathbb{C}^{d+1}$ and can be written in the form :

$$
\forall x, y \in \mathbb{C}^{d+1}, \Lambda_{\alpha, d}(x, y)=a_{\alpha} e^{-i\left\langle x^{\prime}, y^{\prime}\right\rangle} \int_{0}^{1}\left(1-t^{2}\right)^{\alpha-\frac{1}{2}} \cos \left(t x_{d+1} y_{d+1}\right) d t
$$


where $x=\left(x^{\prime}, x_{d+1}\right), x^{\prime}=\left(x_{1}, x_{2}, \ldots, x_{d}\right)$ and $a_{\alpha}$ is the constant given by the relation (2).

The following result summarizes some of the Weinstein kernel's properties.

Proposition 2.

i) For all $\lambda, z \in \mathbb{C}^{d+1}$ and $t \in \mathbb{R}$, we have

$$
\Lambda_{\alpha, d}(\lambda, 0)=1, \Lambda_{\alpha, d}(\lambda, z)=\Lambda_{\alpha, d}(z, \lambda) \text { and } \Lambda_{\alpha, d}(\lambda, t z)=\Lambda_{\alpha, d}(t \lambda, z) .
$$

ii) For all $\nu \in \mathbb{N}^{d+1}, x \in \mathbb{R}_{+}^{d+1}$ and $z \in \mathbb{C}^{d+1}$, we have

$$
\left|D_{z}^{\nu} \Lambda_{\alpha, d}(x, z)\right| \leq\|x\|^{|\nu|} \exp (\|x\|\|\operatorname{Im} z\|)
$$

where $D_{z}^{\nu}=\frac{\partial^{\nu}}{\partial z_{1}^{\nu 1} \ldots \partial z_{d+1}^{\nu d+1}}$ and $|\nu|=\nu_{1}+\ldots+\nu_{d+1}$. In particular

$$
\forall x, y \in \mathbb{R}_{+}^{d+1},\left|\Lambda_{\alpha, d}(x, y)\right| \leq 1 .
$$

Notations. In what follows, we need the following notations:

- $C_{*}\left(\mathbb{R}^{d+1}\right)$, the space of continuous functions on $\mathbb{R}^{d+1}$, even with respect to the last variable.

- $C_{*, c}\left(\mathbb{R}^{d+1}\right)$, the space of continuous functions on $\mathbb{R}^{d+1}$ with compact support, even with respect to the last variable.

- $C_{*}^{p}\left(\mathbb{R}^{d+1}\right)$, the space of functions of class $C^{p}$ on $\mathbb{R}^{d+1}$, even with respect to the last variable.

- $\mathcal{E}_{*}\left(\mathbb{R}^{d+1}\right)$, the space of $C^{\infty}$-functions on $\mathbb{R}^{d+1}$, even with respect to the last variable.

- $\mathcal{S}_{*}\left(\mathbb{R}^{d+1}\right)$, the Schwartz space of rapidly decreasing functions on $\mathbb{R}^{d+1}$, even with respect to the last variable.

- $\mathcal{D}_{*}\left(\mathbb{R}^{d+1}\right)$, the space of $C^{\infty}$-functions on $\mathbb{R}^{d+1}$ which are of compact support, even with respect to the last variable.

- $L_{\alpha}^{p}\left(\mathbb{R}_{+}^{d+1}\right), 1 \leq p \leq+\infty$, the space of measurable functions on $\mathbb{R}_{+}^{d+1}$ such that

$$
\begin{aligned}
\|f\|_{\alpha, \infty} & =\operatorname{ess} \sup _{x \in \mathbb{R}_{+}^{d+1}}|f(x)|<+\infty \\
\|f\|_{\alpha, p} & =\left[\int_{\mathbb{R}_{+}^{d+1}}|f(x)|^{p} d \mu_{\alpha, d}(x)\right]^{\frac{1}{p}}<+\infty, \text { if } 1 \leq p<+\infty,
\end{aligned}
$$

where $\mu_{\alpha, d}$ is the measure on $\mathbb{R}_{+}^{d+1}$ given by :

$$
d \mu_{\alpha, d}(x)=C_{\alpha, d} x_{d+1}^{2 \alpha+1} d x
$$


$d x$ is the Lebesgue measure on $\mathbb{R}^{d+1}$ and $C_{\alpha, d}$ is the constant given by

$$
C_{\alpha, d}=\frac{1}{(2 \pi)^{\frac{d}{2}} 2^{\alpha} \Gamma(\alpha+1)} .
$$

- $\mathcal{H}_{*}\left(\mathbb{C}^{d+1}\right)$, the space of entire functions on $\mathbb{C}^{d+1}$, even with respect to the last variable, rapidly decreasing and of exponential type.

Definition 1. The Weinstein transform is given for $f \in L_{\alpha}^{1}\left(\mathbb{R}_{+}^{d+1}\right)$ by

$$
\forall \lambda \in \mathbb{R}_{+}^{d+1}, \mathcal{F}_{W}^{\alpha, d}(f)(\lambda)=\int_{\mathbb{R}_{+}^{d+1}} f(x) \Lambda_{\alpha, d}(x, \lambda) d \mu_{\alpha, d}(x),
$$

where $\mu_{\alpha, d}$ is the measure on $\mathbb{R}_{+}^{d+1}$ given by the relation (8).

Using the properties of the classical Fourier transform on $\mathbb{R}^{d}$ and of the Bessel transform, one can easily see the following relation, which will play an important role in the sequel.

Example 1. Let $\phi_{t}, t>0$, be the function defined by :

$$
\forall x \in \mathbb{R}^{d+1}, \phi_{t}(x)=\frac{1}{(2 t)^{\alpha+\frac{d}{2}+1}} e^{-\frac{\|x\|^{2}}{4 t}} .
$$

Then the Weinstein transform $\mathcal{F}_{W}^{\alpha, d}$ of $\phi_{t}$ is given by :

$$
\forall \lambda \in \mathbb{R}_{+}^{d+1}, \mathcal{F}_{W}^{\alpha, d}\left(\phi_{t}\right)(\lambda)=e^{-t\|\lambda\|^{2}}
$$

Some basic properties of the transform $\mathcal{F}_{W}^{\alpha, d}$ are summarized in the following results. For the proofs, we refer to $[4,5,6]$.

Proposition 3.

i) For all $f \in L_{\alpha}^{1}\left(\mathbb{R}_{+}^{d+1}\right)$, we have

$$
\left\|\mathcal{F}_{W}^{\alpha, d}(f)\right\|_{\alpha, \infty} \leq\|f\|_{\alpha, 1} .
$$

ii) For $m \in \mathbb{N}$ and $f \in \mathcal{S}_{*}\left(\mathbb{R}^{d+1}\right)$, we have

$$
\forall y \in \mathbb{R}_{+}^{d+1}, \mathcal{F}_{W}^{\alpha, d}\left[\left(\triangle_{W}^{\alpha, d}\right)^{m} f\right](y)=(-1)^{m}\|y\|^{2 m} \mathcal{F}_{W}^{\alpha, d}(f)(y) .
$$

iii) For all $f$ in $\mathcal{S}_{*}\left(\mathbb{R}^{d+1}\right)$ and $m \in \mathbb{N}$, we have

$$
\forall \lambda \in \mathbb{R}_{+}^{d+1},\left(\triangle_{W}^{\alpha, d}\right)^{m}\left[\mathcal{F}_{W}^{\alpha, d}(f)\right](\lambda)=\mathcal{F}_{W}^{\alpha, d}\left(P_{m} f\right)(\lambda),
$$

where $P_{m}(\lambda)=(-1)^{m}\|\lambda\|^{2 m}$. 
Theorem 1.

i) The Weinstein transform $\mathcal{F}_{W}^{\alpha, d}$ is a topological isomorphism from $\mathcal{S}_{*}\left(\mathbb{R}^{d+1}\right)$ onto itself and from $\mathcal{D}_{*}\left(\mathbb{R}^{d+1}\right)$ onto $\mathcal{H}_{*}\left(\mathbb{C}^{d+1}\right)$.

ii) Let $f \in \mathcal{S}_{*}\left(\mathbb{R}^{d+1}\right)$. The inverse transform $\left(\mathcal{F}_{W}^{\alpha, d}\right)^{-1}$ is given by

$$
\forall x \in \mathbb{R}_{+}^{d+1},\left(\mathcal{F}_{W}^{\alpha, d}\right)^{-1}(f)(x)=\mathcal{F}_{W}^{\alpha, d}(f)(-x)
$$

iii) Let $f \in L_{\alpha}^{1}\left(\mathbb{R}_{+}^{d+1}\right)$. If $\mathcal{F}_{W}^{\alpha, d}(f) \in L_{\alpha}^{1}\left(\mathbb{R}_{+}^{d+1}\right)$, then we have

$$
f(x)=\int_{\mathbb{R}_{+}^{d+1}} \mathcal{F}_{W}^{\alpha, d}(f)(y) \Lambda_{\alpha, d}(-x, y) d \mu_{\alpha, d}(y), \text { a.e } x \in \mathbb{R}_{+}^{d+1} .
$$

Theorem 2.

i) For all $f, g \in \mathcal{S}_{*}\left(\mathbb{R}^{d+1}\right)$, we have the following Parseval formula

$$
\int_{\mathbb{R}_{+}^{d+1}} f(x) \overline{g(x)} d \mu_{\alpha, d}(x)=\int_{\mathbb{R}_{+}^{d+1}} \mathcal{F}_{W}^{\alpha, d}(f)(\lambda) \overline{\mathcal{F}_{W}^{\alpha, d}(g)(\lambda)} d \mu_{\alpha, d}(\lambda) .
$$

ii) ( Plancherel formula).

For all $f \in \mathcal{S}_{*}\left(\mathbb{R}^{d+1}\right)$, we have :

$$
\int_{\mathbb{R}_{+}^{d+1}}|f(x)|^{2} d \mu_{\alpha, d}(x)=\int_{\mathbb{R}_{+}^{d+1}}\left|\mathcal{F}_{W}^{\alpha, d}(f)(\lambda)\right|^{2} d \mu_{\alpha, d}(\lambda) .
$$

iii) ( Plancherel Theorem ) :

The transform $\mathcal{F}_{W}^{\alpha, d}$ extends uniquely to an isometric isomorphism on $L_{\alpha}^{2}\left(\mathbb{R}_{+}^{d+1}\right)$.

Definition 2. The translation operator $T_{x}, x \in \mathbb{R}_{+}^{d+1}$, associated with the Weinstein operator $\Delta_{W}^{\alpha, d}$ is defined on $C_{*}\left(\mathbb{R}^{d+1}\right)$, for all $y \in \mathbb{R}_{+}^{d+1}$, by :

$$
T_{x} f(y)=\frac{a_{\alpha}}{2} \int_{0}^{\pi} f\left(x^{\prime}+y^{\prime}, \sqrt{x_{d+1}^{2}+y_{d+1}^{2}+2 x_{d+1} y_{d+1} \cos \theta}\right)(\sin \theta)^{2 \alpha} d \theta
$$

where $x^{\prime}+y^{\prime}=\left(x_{1}+y_{1}, \ldots, x_{d}+y_{d}\right)$ and $a_{\alpha}$ is the constant given by (2).

Example 2. Let $\phi_{t}, t>0$, be the function given by the relation (11). Then for all $x, y \in \mathbb{R}_{+}^{d+1}$, we have

$$
T_{x}\left(\phi_{t}\right)(y)=\frac{1}{(2 t)^{\alpha+\frac{d}{2}+1}} e^{-\frac{\|x\|^{2}+\|y\|^{2}}{4 t}} \Lambda_{\alpha, d}\left(x,-i \frac{y}{2 t}\right) .
$$

The following proposition summarizes some properties of the Weinstein translation operator. 


\section{Proposition 4.}

i) For $f \in C_{*}\left(\mathbb{R}^{d+1}\right)$, we have

$$
\forall x, y \in \mathbb{R}_{+}^{d+1}, T_{x} f(y)=T_{y} f(x) \text { and } T_{0} f=f .
$$

ii) For all $f \in \mathcal{E}_{*}\left(\mathbb{R}^{d+1}\right)$ and $y \in \mathbb{R}_{+}^{d+1}$, the function $x \mapsto T_{x} f(y)$ belongs to $\varepsilon_{*}\left(\mathbb{R}^{d+1}\right)$.

iii) We have

$$
\forall x \in \mathbb{R}_{+}^{d+1}, \Delta_{W}^{\alpha, d} \circ T_{x}=T_{x} \circ \Delta_{W}^{\alpha, d} .
$$

iv) Let $f \in L_{\alpha}^{p}\left(\mathbb{R}_{+}^{d+1}\right), 1 \leq p \leq+\infty$ and $x \in \mathbb{R}_{+}^{d+1}$. Then $T_{x} f$ belongs to $L_{\alpha}^{p}\left(\mathbb{R}_{+}^{d+1}\right)$ and we have

$$
\left\|T_{x} f\right\|_{\alpha, p} \leq\|f\|_{\alpha, p} .
$$

v) The function $\Lambda_{\alpha, d}(., \lambda), \lambda \in \mathbb{C}^{d+1}$, satisfies on $\mathbb{R}_{+}^{d+1}$ the following product formula:

$$
\forall y \in \mathbb{R}_{+}^{d+1}, \Lambda_{\alpha, d}(x, \lambda) \Lambda_{\alpha, d}(y, \lambda)=T_{x}\left[\Lambda_{\alpha, d}(., \lambda)\right](y) .
$$

vi) Let $f \in L_{\alpha}^{p}\left(\mathbb{R}_{+}^{d+1}\right), p=1$ or 2 and $x \in \mathbb{R}_{+}^{d+1}$, we have

$$
\forall y \in \mathbb{R}_{+}^{d+1}, \mathcal{F}_{W}^{\alpha, d}\left(T_{x} f\right)(y)=\Lambda_{\alpha, d}(x, y) \mathcal{F}_{W}^{\alpha, d}(f)(y) .
$$

vii) The space $\mathcal{S}_{*}\left(\mathbb{R}^{d+1}\right)$ is invariant under the operators $T_{x}, x \in \mathbb{R}_{+}^{d+1}$.

Definition 3. The Weinstein convolution product of $f, g \in L_{\alpha}^{1}\left(\mathbb{R}_{+}^{d+1}\right)$ is given by:

$$
\forall x \in \mathbb{R}_{+}^{d+1}, f *_{W} g(x)=\int_{\mathbb{R}_{+}^{d+1}} T_{x} f(-y) g(y) d \mu_{\alpha, d}(y) .
$$

\section{Proposition 5.}

i) Let $p, q, r \in[1,+\infty]$ such that $\frac{1}{p}+\frac{1}{q}-\frac{1}{r}=1$.

Then for all $f \in L_{\alpha}^{p}\left(\mathbb{R}_{+}^{d+1}\right)$ and $g \in L_{\alpha}^{q}\left(\mathbb{R}_{+}^{d+1}\right)$, the function $f *_{W} g$ belongs to $L_{\alpha}^{r}\left(\mathbb{R}_{+}^{d+1}\right)$ and we have

$$
\left\|f *_{W} g\right\|_{\alpha, r} \leq\|f\|_{\alpha, p}\|g\|_{\alpha, q} .
$$

ii) For all $f, g \in L_{\alpha}^{1}\left(\mathbb{R}_{+}^{d+1}\right), \quad\left(\right.$ resp. $\left.\mathcal{S}_{*}\left(\mathbb{R}^{d+1}\right)\right), \quad f *_{W} g \in L_{\alpha}^{1}\left(\mathbb{R}_{+}^{d+1}\right)$ $\left(\right.$ resp. $\left.\mathcal{S}_{*}\left(\mathbb{R}^{d+1}\right)\right)$ and we have

$$
\mathcal{F}_{W}^{\alpha, d}\left(f *_{W} g\right)=\mathcal{F}_{W}^{\alpha, d}(f) \mathcal{F}_{W}^{\alpha, d}(g) .
$$




\section{The Weinstein intertwining operator and its dual}

\section{Definition 4.}

i) The Weinstein intertwining operator is the operator $\mathcal{R}_{W}^{\alpha, d}$ defined on $C_{*}\left(\mathbb{R}^{d+1}\right)$ by :

$$
\forall x \in \mathbb{R}_{+}^{d+1}, \mathcal{R}_{W}^{\alpha, d}(f)(x)=a_{\alpha} \int_{0}^{1}\left(1-t^{2}\right)^{\alpha-\frac{1}{2}} f\left(x^{\prime}, t x_{d+1}\right) d t,
$$

where $a_{\alpha}$ is the constant given by the relation (2)

ii) The dual of the Weinstein intertwining operator is defined on $\mathcal{D}_{*}\left(\mathbb{R}^{d+1}\right)$, for all $y=\left(y^{\prime}, y_{d+1}\right) \in \mathbb{R}_{+}^{d+1}$ by:

$$
{ }^{t} \mathcal{R}_{W}^{\alpha, d}(f)(y)=a_{\alpha} \int_{y_{d+1}}^{+\infty}\left(s^{2}-y_{d+1}^{2}\right)^{\alpha-\frac{1}{2}} f\left(y^{\prime}, s\right) s d s .
$$

Remark 2. For all $x=\left(x^{\prime}, x_{d+1}\right), y=\left(y^{\prime}, y_{d+1}\right) \in \mathbb{R}_{+}^{d+1}$, we have

$$
\mathcal{R}_{W}^{\alpha, d}\left(e^{-i\left\langle x^{\prime}, .\right\rangle} \cos \left(x_{d+1} .\right)\right)(y)=\Lambda_{\alpha, d}(x, y) .
$$

Proposition 6.

i) $\mathcal{R}_{W}^{\alpha, d}$ is a topological isomorphism from $\mathcal{E}_{*}\left(\mathbb{R}_{+}^{d+1}\right)$ onto itself satisfying the following transmutation relation

$$
\triangle_{W}^{\alpha, d}\left(\mathcal{R}_{W}^{\alpha, d} f\right)=\mathcal{R}_{W}^{\alpha, d}\left(\triangle_{d+1} f\right), f \in \mathcal{E}_{*}\left(\mathbb{R}_{+}^{d+1}\right),
$$

where $\triangle_{d+1}=\sum_{i=1}^{d+1} \frac{\partial^{2}}{\partial x_{i}^{2}}$ is the Laplacian operator on $\mathbb{R}^{d+1}$.

ii) ${ }^{t} \mathcal{R}_{W}^{\alpha, d}$ can be extended to a topological isomorphism from $\mathcal{S}_{*}\left(\mathbb{R}^{d+1}\right)$ onto itself and satisfies the following transmutation relation

$$
{ }^{t} \mathcal{R}_{W}^{\alpha, d}\left(\triangle_{W}^{\alpha, d} f\right)=\triangle_{d+1}\left({ }^{t} \mathcal{R}_{W}^{\alpha, d} f\right), f \in \mathcal{S}_{*}\left(\mathbb{R}^{d+1}\right) .
$$

Example 3. Let $\beta \in \mathbb{R}, \beta>0$ and $f_{\beta}$ be the function defined on $\mathbb{R}_{+}^{d+1}$ by

$$
\forall x=\left(x^{\prime}, x_{d+1}\right) \in \mathbb{R}_{+}^{d+1}, f_{\beta}(x)=\frac{\sqrt{\pi} \Gamma(\alpha+\beta+1)}{\Gamma(\alpha+1) \Gamma\left(\beta+\frac{1}{2}\right)} x_{d+1}^{2 \beta} .
$$

Then, we have

$$
\mathcal{R}_{W}^{\alpha, d}\left(f_{\beta}\right)=f_{\beta} .
$$

Proposition 7. For all $f \in \mathcal{S}_{*}\left(\mathbb{R}^{d+1}\right)$, we have

$$
\forall y \in \mathbb{R}_{+}^{d+1}, \mathcal{F}_{W}^{\alpha, d}(f)(y)=\mathcal{F}_{o} \circ{ }^{t} \mathcal{R}_{W}^{\alpha, d}(f)(y),
$$


where $\mathcal{F}_{o}$ is the classical Fourier transform defined for $f \in C_{*, c}\left(\mathbb{R}^{d+1}\right)$ by

$$
\forall y \in \mathbb{R}_{+}^{d+1}, \mathcal{F}_{o}(f)(y)=C_{\alpha, d} \int_{\mathbb{R}_{+}^{d+1}} f(x) e^{-i\left\langle y^{\prime}, x^{\prime}\right\rangle} \cos \left(x_{d+1} y_{d+1}\right) d x
$$

and $C_{\alpha, d}$ is the constant given by the relation (9).

Proposition 8. For all $f \in \mathcal{S}_{*}\left(\mathbb{R}^{d+1}\right)$ and $g \in \mathcal{D}_{*}\left(\mathbb{R}^{d+1}\right)$, we have

$$
{ }^{t} \mathcal{R}_{W}^{\alpha, d}\left(f *_{W} g\right)={ }^{t} \mathcal{R}_{W}^{\alpha, d}(f) *{ }^{t} \mathcal{R}_{W}^{\alpha, d}(g),
$$

where $*$ is the clssical convolution product given by :

$$
\forall x \in \mathbb{R}^{d+1}, f * g(x)=\int_{\mathbb{R}^{d+1}} f(x-y) g(y) d y .
$$

Proposition 9. The operators $\mathcal{R}_{W}^{\alpha, d}$ and ${ }^{t} \mathcal{R}_{W}^{\alpha, d}$ are linked by the following relation : for $f \in \mathcal{C}_{*}\left(\mathbb{R}^{d+1}\right)$ and $g \in \mathcal{D}_{*}\left(\mathbb{R}^{d+1}\right)$,

$$
\int_{\mathbb{R}_{+}^{d+1}} \mathcal{R}_{W}^{\alpha, d}(f)(y) g(x) x_{d+1}^{2 \alpha+1} d x=\int_{\mathbb{R}_{+}^{d+1}}{ }^{t} \mathcal{R}_{W}^{\alpha, d}(g)(y) f(y) d y .
$$

\section{Inversion formulas for the Weinstein intertwining op- erator and its dual}

In this section, we show that the Weinstein intertwining operator and its dual are bijective on spaces other than $\mathcal{E}_{*}\left(\mathbb{R}_{+}^{d+1}\right)$ and $\mathcal{D}_{*}\left(\mathbb{R}^{d+1}\right)$ and we give inversion formulas for these operators.

We consider the operators $\mathcal{K}$ and $\mathcal{K}_{W}$ defined by :

$$
\forall x \in \mathbb{R}_{+}^{d+1}, \mathcal{K}(f)(x)=\mathcal{F}_{0}^{-1}\left[\xi_{\alpha} \mathcal{F}_{0}(f)\right](x)
$$

and

$$
\forall x \in \mathbb{R}_{+}^{d+1}, \mathcal{K}_{\mathcal{W}}(f)(x)=\left(\mathcal{F}_{W}^{\alpha, d}\right)^{-1}\left[\xi_{\alpha} \mathcal{F}_{W}^{\alpha, d}(f)\right](x),
$$

where

$$
\forall x \in \mathbb{R}_{+}^{d+1}, \xi_{\alpha}(x)=\frac{\pi}{2^{2 \alpha+1}(\Gamma(\alpha+1))^{2}} x_{d+1}^{2 \alpha+1} .
$$

Notations. In what follows, we need the following notations:

- $\mathcal{S}_{*}^{0}\left(\mathbb{R}^{d+1}\right)$ is the subspace of $\mathcal{S}_{*}\left(\mathbb{R}^{d+1}\right)$ consisting of functions $f$ such that

$$
\forall \nu=\left(\nu_{1}, \ldots, \nu_{d+1}\right) \in \mathbb{N}^{d+1}, \quad D^{\nu} f(0)=0,
$$


where $D^{\nu}=\frac{\partial^{|\nu|}}{\partial x_{1}^{\nu_{1}} \ldots \partial x_{d+1}^{\nu_{d+1}}}$ and $|\nu|=\nu_{1}+\ldots+\nu_{d+1}$.

- $\mathcal{S}_{*, 0}\left(\mathbb{R}^{d+1}\right)$ is the subspace of $\mathcal{S}_{*}\left(\mathbb{R}^{d+1}\right)$ consisting of functions $f$ such that for all $\nu \in \mathbb{N}^{d+1}$,

$$
\int_{\mathbb{R}_{+}^{d+1}} f(x) x^{\nu} d x=0,
$$

where for $\nu=\left(\nu_{1}, \ldots, \nu_{d+1}\right) \in \mathbb{N}^{d+1}$ and $x=\left(x_{1}, \ldots, x_{d+1}\right) \in \mathbb{R}^{d+1}$, we have $x^{\nu}=x_{1}^{\nu_{1}} \ldots x_{d+1}^{\nu_{d+1}}$.

- $\mathcal{S}_{*, 0}^{0}\left(\mathbb{R}^{d+1}\right)$ is the subspace of $\mathcal{S}_{*}\left(\mathbb{R}^{d+1}\right)$ consisting of functions $f$ such that for all $\nu \in \mathbb{N}^{d+1}$,

$$
\int_{\mathbb{R}_{+}^{d+1}} f(x) m_{\nu}(x) d \mu_{\alpha, d}(x)=0,
$$

where for $\nu=\left(\nu_{1}, \ldots, \nu_{d+1}\right) \in \mathbb{N}^{d+1}$ and $x \in \mathbb{R}^{d+1}$, we have

$$
m_{\nu}=\mathcal{R}_{W}^{\alpha, d}\left(\frac{u^{\nu}}{\nu !}\right)(x)
$$

and $\nu !=\nu_{1} ! \nu_{2} ! \ldots \nu_{d+1} !$

Theorem 3. The transform ${ }^{t} \mathcal{R}_{W}^{\alpha, d}$ is a topological automorphism from $\mathcal{S}_{*, 0}^{0}\left(\mathbb{R}^{d+1}\right)$ onto $\mathcal{S}_{*, 0}\left(\mathbb{R}^{d+1}\right)$.

Proposition 10.

i) For all $f$ in $\mathcal{S}_{*, 0}\left(\mathbb{R}^{d+1}\right)$ and $g$ in $\mathcal{S}_{*}\left(\mathbb{R}^{d+1}\right)$, the function $f * g$ belongs to $\mathcal{S}_{*, 0}\left(\mathbb{R}^{d+1}\right)$.

ii) For all $f$ in $\mathcal{S}_{*, 0}^{0}\left(\mathbb{R}^{d+1}\right)$ and $g$ in $\mathcal{S}_{*}\left(\mathbb{R}^{d+1}\right)$, the function $f *_{W} g$ belongs to $\mathcal{S}_{*, 0}^{0}\left(\mathbb{R}^{d+1}\right)$.

Proof. We deduce these results from the relation (25), Theorem 1 and the properties of the classical Fourier transform $\mathcal{F}_{0}$.

\section{Proposition 11.}

i) The operator $\mathcal{K}$, ( respectively $\mathcal{K}_{W}$ ), is a topological automorphism of $\mathcal{S}_{*, 0}\left(\mathbb{R}^{d+1}\right)$, (respectively $\left.\mathcal{S}_{*, 0}^{0}\left(\mathbb{R}^{d+1}\right)\right)$.

ii) For all $f$ in $\mathcal{S}_{*, 0}^{0}\left(\mathbb{R}^{d+1}\right)$, we have :

$$
\mathcal{K}_{W}(f)=\left({ }^{t} \mathcal{R}_{W}^{\alpha, d}\right)^{-1} \circ \mathcal{K} \circ{ }^{t} \mathcal{R}_{W}^{\alpha, d} .
$$

Proof. i) The mapping $f \rightarrow \xi_{\alpha} f$ is a topological automorphism of $\mathcal{S}_{*}^{0}\left(\mathbb{R}^{d+1}\right)$. We deduce the result from Theorem 1 and the fact that $\mathcal{F}_{0}$ is a topological automorphism from $\mathcal{S}_{*, 0}\left(\mathbb{R}^{d+1}\right)$ onto $\mathcal{S}_{*}^{0}\left(\mathbb{R}^{d+1}\right)$.

ii) We obtain the result from the relations (37) and (31), and Theorem 3. 
Proposition 12. i) For all $f \in \mathcal{S}_{*, 0}\left(\mathbb{R}^{d+1}\right)$ and $g \in \mathcal{S}_{*}\left(\mathbb{R}^{d+1}\right)$, we have

$$
\mathcal{K}(f * g)=\mathcal{K}(f) * g .
$$

ii) For all $f \in \mathcal{S}_{*, 0}^{0}\left(\mathbb{R}^{d+1}\right)$ and $g \in \mathcal{S}_{*}\left(\mathbb{R}^{d+1}\right)$, we have

$$
\mathcal{K}_{W}\left(f *_{W} g\right)=\mathcal{K}_{W}(f) *_{W} g .
$$

Proof. We obtain these relations from (36), (37), Proposition 9, the relation (25), and the properties of the classical transform $\mathcal{F}_{0}$ and of the classical convolution product on $\mathbb{R}^{d+1}$.

Theorem 4. We have the following inversion formulas for the operators $\mathcal{R}_{W}^{\alpha, d}$ and ${ }^{t} \mathcal{R}_{W}^{\alpha, d}$.

i) For all $f \in \mathcal{S}_{*, 0}^{0}\left(\mathbb{R}^{d+1}\right)$, we have

$$
f=\mathcal{R}_{W}^{\alpha, d} \mathcal{K}^{t} \mathcal{R}_{W}^{\alpha, d}(f) .
$$

ii) For all $f \in \mathcal{S}_{*, 0}\left(\mathbb{R}^{d+1}\right)$, we have

$$
f={ }^{t} \mathcal{R}_{W}^{\alpha, d} \mathcal{K}_{W} \mathcal{R}_{W}^{\alpha, d}(f) .
$$

iii) For all $f \in \mathcal{S}_{*, 0}\left(\mathbb{R}^{d+1}\right)$, we have

$$
f=\mathcal{K}^{t} \mathcal{R}_{W}^{\alpha, d} \mathcal{R}_{W}^{\alpha, d}(f) .
$$

iv) For all $f \in \mathcal{S}_{*, 0}^{0}\left(\mathbb{R}^{d+1}\right)$, we have

$$
f=\mathcal{K}_{W} \mathcal{R}_{W}^{\alpha, d}{ }^{t} \mathcal{R}_{W}^{\alpha, d}(f) .
$$

Proof. i) Let $f$ be in $\mathcal{S}_{*, 0}^{0}\left(\mathbb{R}^{d+1}\right)$. Using Theorem 1, the relations (28) and $(25)$, and the inversion formula for the classical Fourier $\mathcal{F}_{0}$, we obtain for all $x \in \mathbb{R}_{+}^{d+1}$,

$$
\begin{aligned}
f(x) & =\int_{\mathbb{R}_{+}^{d+1}} \mathcal{F}_{W}^{\alpha, d}(f)(y) \Lambda_{\alpha, d}(-x, y) d \mu_{\alpha, d}(y) \\
& =\int_{\mathbb{R}_{+}^{d+1}} \mathcal{F}_{W}^{\alpha, d}(f)(y) \mathcal{R}_{W}^{\alpha, d}\left(e^{i\left\langle., y^{\prime}\right\rangle} \cos \left(. y_{d+1}\right)\right)(x) d \mu_{\alpha, d}(y) \\
& =\mathcal{R}_{W}^{\alpha, d}\left(\int_{\mathbb{R}_{+}^{d+1}} \mathcal{F}_{W}^{\alpha, d}(f)(y) e^{i\left\langle\cdot, y^{\prime}\right\rangle} \cos \left(. y_{d+1}\right) d \mu_{\alpha, d}(y)\right)(x) \\
& =\mathcal{R}_{W}^{\alpha, d}\left(\int_{\mathbb{R}_{+}^{d+1}} \mathcal{F}_{0} o^{t} \mathcal{R}_{W}^{\alpha, d}(f)(y) e^{i\left\langle., y^{\prime}\right\rangle} \cos \left(. y_{d+1}\right) d \mu_{\alpha, d}(y)\right)(x) \\
& =\mathcal{R}_{W}^{\alpha, d} \mathcal{F}_{0}^{-1}\left(\xi_{\alpha} \mathcal{F}_{0} o^{t} \mathcal{R}_{W}^{\alpha, d}\right)(f)(x) \\
& =\mathcal{R}_{W}^{\alpha, d} \mathcal{K}^{t} \mathcal{R}_{W}^{\alpha, d}(f)(x) .
\end{aligned}
$$


ii) From the relation (42) and the relation (25), we obtain

$$
\forall f \in \mathcal{S}_{*, 0}\left(\mathbb{R}^{d+1}\right), f={ }^{t} \mathcal{R}_{W}^{\alpha, d} \mathcal{K}_{W} \mathcal{R}_{W}^{\alpha, d}(f) .
$$

iii) We obtain the relation (44) by writing the relation (42) for the function $\mathcal{R}_{W}^{\alpha, d}(f)$.

iv) We obtain the relation (45) by writing the relation (43) for the function ${ }^{t} \mathcal{R}_{W}^{\alpha, d}(f)$.

Corollary 1. The operator $\mathcal{R}_{W}^{\alpha, d}$ is a topological isomorphism from $\mathcal{S}_{*, 0}\left(\mathbb{R}^{d+1}\right)$ onto $\mathcal{S}_{*, 0}^{0}\left(\mathbb{R}^{d+1}\right)$.

Proof. We deduce the result from Proposition 10, i), Theorem 3 and the relation (42)

Corollary 2. For all $f$ in $\mathcal{S}_{*, 0}\left(\mathbb{R}^{d+1}\right)$ and $g$ in $\mathcal{S}\left(\mathbb{R}^{d+1}\right)$, we have

$$
\mathcal{R}_{W}^{\alpha, d}(f * g)=\mathcal{R}_{W}^{\alpha, d}(f) * W\left({ }^{t} \mathcal{R}_{W}^{\alpha, d}\right)^{-1}(g) .
$$

Proof. Using the relations (33), (42), (40) and (44), we obtain

$$
\begin{gathered}
\left(\mathcal{R}_{W}^{\alpha, d}\right)^{-1}\left[\mathcal{R}_{W}^{\alpha, d}(f) *{ }_{W}\left({ }^{t} \mathcal{R}_{W}^{\alpha, d}\right)^{-1}(g)\right]=\mathcal{K}^{t} \mathcal{R}_{W}^{\alpha, d}\left[\mathcal{R}_{W}^{\alpha, d}(f) *{ }_{W}\left({ }^{t} \mathcal{R}_{W}^{\alpha, d}\right)^{-1}(g)\right] \\
=\mathcal{K}\left[{ }^{t} \mathcal{R}_{W}^{\alpha, d} \mathcal{R}_{W}^{\alpha, d}(f) * g\right]=\left[\mathcal{K}^{t} \mathcal{R}_{W}^{\alpha, d} \mathcal{R}_{W}^{\alpha, d}(f)\right] * g=f * g
\end{gathered}
$$

Thus, we obtain the result from Corollary 1.

\section{$5 \quad$ Weinstein Wavelets and Weinstein Wavelet transform}

Definition 5. A measurable function $g$ on $\mathbb{R}_{+}^{d+1}$ is a classical wavelet on $\mathbb{R}_{+}^{d+1}$ if it satisfies, for almost all $x \in \mathbb{R}_{+}^{d+1}$, the condition :

$$
0<C_{g}^{0}=\int_{0}^{\infty}\left|\mathcal{F}_{0}(g)(\lambda x)\right|^{2} \frac{d \lambda}{\lambda}<\infty,
$$

where $\mathcal{F}_{0}$ is the classical Fourier transform given by the relation (31).

Notations. We denote by :

- $L^{1}\left(\mathbb{R}^{d+1}\right)$, the space of integrable functions on $\mathbb{R}^{d+1}$ with respect to the Lebesgue measure.

- $L^{2}\left(\mathbb{R}^{d+1}\right)$, the space of square integrable functions on $\mathbb{R}^{d+1}$ with respect to the Lebesgue measure.

- $g_{a, x}, a>0, x \in \mathbb{R}^{d+1}$, the family of classical wavelets on $\mathbb{R}^{d+1}$ in $L^{2}\left(\mathbb{R}^{d+1}\right)$ defined by :

$$
\forall y \in \mathbb{R}^{d+1}, g_{a, x}(y)=H_{a}(g)(x-y)
$$


where $H_{a}$ is the dilatation operator given by :

$$
\forall x \in \mathbb{R}^{d+1}, H_{a}(g)(x)=g_{a}^{0}(x)=\frac{1}{a^{d+1}} g\left(\frac{x}{a}\right) .
$$

Definition 6. Let $g$ be a classical Wavelet on $\mathbb{R}^{d+1}$ in $L^{2}\left(\mathbb{R}^{d+1}\right)$. The classical continuous Wavelet transform $S_{g}$ on $\mathbb{R}^{d+1}$ is defined for regular functions $f$ on $\mathbb{R}^{d+1}$ by :

$$
\forall x \in \mathbb{R}^{d+1}, S_{g}(f)(a, x)=\int_{\mathbb{R}^{d+1}} f(y) \overline{g_{a, x}(y)} d y,
$$

where $g_{a, x}, a>0, x \in \mathbb{R}^{d+1}$ are the family given by the relation (48).

Remark 3. The transform $S_{g}$ can also be written in the form:

$$
S_{g}(f)(a, x)=f * \overline{g_{a}^{0}}(x),
$$

where $*$ is the clssical convolution product given by the relation (34).

The transform $S_{g}$ has been introduced in [10]. Various properties of this transform were studied by many anthors (see [10] and [16]). In particular, we have the following results.

Proposition 13. i)( Plancherel formula) For all $f \in L^{2}\left(\mathbb{R}^{d+1}\right)$ we have :

$$
\int_{\mathbb{R}^{d+1}}|f(x)|^{2} d \mu_{\alpha, d}(x)=\frac{1}{C_{g}^{0}} \int_{0}^{\infty} \int_{\mathbb{R}^{d+1}}\left|S_{g}(f)(a, x)\right|^{2} d x \frac{d a}{a} .
$$

ii)( Inversion formula) For all $f$ in $L^{1}\left(\mathbb{R}^{d+1}\right)$ such that $\mathcal{F}_{0}(f)$ belongs to $L^{1}\left(\mathbb{R}^{d+1}\right)$, we have

$$
f(x)=\frac{1}{C_{g}^{0}} \int_{0}^{\infty}\left(\int_{\mathbb{R}^{d+1}} S_{g}(f)(a, y) g_{a, x}(y) d y\right) \frac{d a}{a}, \text { a.e } x \in \mathbb{R}^{d+1} .
$$

Here, the inner integral and the outer integral are absolutely convergent, but possibly not the double integral.

Definition 7. A Weinstein Wavelet on $\mathbb{R}_{+}^{d+1}$ is a measurable function $g$ on $\mathbb{R}_{+}^{d+1}$ satisfying for almost all $x \in \mathbb{R}_{+}^{d+1}$, the condition :

$$
0<C_{g}=\int_{0}^{\infty}\left|\mathcal{F}_{W}^{\alpha, d}(g)(\lambda x)\right|^{2} \frac{d \lambda}{\lambda}<\infty
$$


Example 4. The function $g_{t}, t>0$, given by :

$$
\forall x \in \mathbb{R}_{+}^{d+1}, g_{t}(x)=-\frac{d}{d t} \phi_{t}(x)
$$

where $\phi_{t}$ is the function given by the relation (11) is a Weinstein Wavelet on $\mathbb{R}_{+}^{d+1}$ and we have $C_{g_{t}}=\frac{1}{8 t^{2}}$.

Proposition 14. A function $g$ is a Weinstein Wavelet on $\mathbb{R}_{+}^{d+1}$ in $\mathcal{S}\left(\mathbb{R}^{d+1}\right)$, respectively $\mathcal{S}_{*, 0}^{0}\left(\mathbb{R}^{d+1}\right)$ if and only if the function ${ }^{t} \mathcal{R}_{W}^{\alpha, d}(g)$ is a classical Wavelet on $\mathbb{R}_{+}^{d+1}$ in $\mathcal{S}\left(\mathbb{R}^{d+1}\right)$, respectively $\mathcal{S}_{*, 0}\left(\mathbb{R}^{d+1}\right)$ and we have

$$
C_{t \mathcal{R}_{W}^{\alpha, d}(g)}^{0}=C_{g} .
$$

Proof. We deduce these results from Proposition 6, Theorem 3 and the relation (31).

Let $a \in] 0,+\infty\left[\right.$ and $g$ be a regular function on $\mathbb{R}^{d+1}$. We consider the function $g_{a}$ given by :

$$
\forall x \in \mathbb{R}^{d+1}, g_{a}(x)=\frac{1}{a^{2 \alpha+d+2}} g\left(\frac{x}{a}\right) .
$$

The function $g_{a}$ satisfies the following properties.

Proposition 15. i) For all $g$ in $L_{\alpha}^{2}\left(\mathbb{R}_{+}^{d+1}\right)$, the function $g_{a}$ belongs to $L_{\alpha}^{2}\left(\mathbb{R}_{+}^{d+1}\right)$ and we have

$$
\forall x \in \mathbb{R}_{+}^{d+1}, \mathcal{F}_{W}^{\alpha, d}\left(g_{a}\right)(x)=\mathcal{F}_{W}^{\alpha, d}(g)(a x) .
$$

ii) For all $g$ in $\mathcal{S}\left(\mathbb{R}^{d+1}\right)$ (respectively $\mathcal{S}_{*, 0}^{0}\left(\mathbb{R}^{d+1}\right)$ ), the function $g_{a}$ belongs to $\mathcal{S}\left(\mathbb{R}^{d+1}\right)$ ( respectively $\mathcal{S}_{*, 0}^{0}\left(\mathbb{R}^{d+1}\right)$ ) and we have

$$
g_{a}=\left({ }^{t} \mathcal{R}_{W}^{\alpha, d}\right)^{-1} \circ H_{a} \circ^{t} \mathcal{R}_{W}^{\alpha, d}(g) .
$$

Definition 8. Let $g$ be a Weinstein Wavelet on $\mathbb{R}_{+}^{d+1}$ in $L_{\alpha}^{2}\left(\mathbb{R}_{+}^{d+1}\right)$. The Weinstein continuous Wavelet transform on $\mathbb{R}_{+}^{d+1}$ is defined for regular functions $f$ on $\mathbb{R}_{+}^{d+1}$ by :

$$
S_{g}^{W}(f)(a, x)=\int_{\mathbb{R}_{+}^{d+1}} f(y) \overline{g_{a, x}(y)} d \mu_{\alpha, d}(y),
$$

where $g_{a, x}, a>0, x \in \mathbb{R}_{+}^{d+1}$ are the family of Weinstein Wavelets on $\mathbb{R}_{+}^{d+1}$ in $L_{\alpha}^{2}\left(\mathbb{R}_{+}^{d+1}\right)$ given by :

$$
\forall y \in \mathbb{R}_{+}^{d+1}, g_{a, x}(y)=T_{x} g_{a}(y) .
$$

Here $T_{x}, x \in \mathbb{R}_{+}^{d+1}$, are the Weinstein translation operators given by the relation (19). 
Remark 4. The transform $S_{g}^{W}$ can also be written in the form

$$
S_{g}^{W}(f)(a, x)=f *_{W} \overline{g_{a}}(x),
$$

where ${ }_{W}$ is the Weinstein convolution product given by the relation (23).

Example 5. We consider the function $\phi_{t}$ given by the relation (11). Using the relation (20), we deduce that the family $g_{a, x}, a>0, x \in \mathbb{R}_{+}^{d+1}$ given by

$$
\forall y \in \mathbb{R}_{+}^{d+1}, g_{a, x}(y)=-T_{x}\left(\frac{d}{d t} \phi_{t}\right)(y)
$$

is a family of Weinstein Wavelet on $\mathbb{R}_{+}^{d+1}$ in $\mathcal{S}_{*}\left(\mathbb{R}^{d+1}\right)$.

Theorem 5. (Plancherel formula for $S_{g}^{W}$ )

For all $f \in L_{\alpha}^{2}\left(\mathbb{R}_{+}^{d+1}\right)$ we have :

$$
\int_{\mathbb{R}_{+}^{d+1}}|f(x)|^{2} d \mu_{\alpha, d}(x)=\frac{1}{C_{g}} \int_{0}^{\infty} \int_{\mathbb{R}_{+}^{d+1}}\left|S_{g}^{W}(f)(a, x)\right|^{2} d \mu_{\alpha, d}(x) \frac{d a}{a} .
$$

Proof. Using Fubini-Tonnelli's theorem, Proposition 9, ii) and the relations (62), (58), we get

$$
\begin{gathered}
\frac{1}{C_{g}} \int_{0}^{\infty} \int_{\mathbb{R}_{+}^{d+1}}\left|S_{g}^{W}(f)(a, x)\right|^{2} d \mu_{\alpha, d}(x) \frac{d a}{a}=\frac{1}{C_{g}} \int_{0}^{\infty}\left(\int_{\mathbb{R}_{+}^{d+1}}\left|f *_{W} \overline{g_{a}}(x)\right|^{2} d \mu_{\alpha, d}(x)\right) \frac{d a}{a} \\
=\frac{1}{C_{g}} \int_{0}^{\infty}\left(\int_{\mathbb{R}_{+}^{d+1}}\left|\mathcal{F}_{W}^{\alpha, d}(f)(x)\right|^{2}\left|\mathcal{F}_{W}^{\alpha, d}\left(\overline{g_{a}}\right)(x)\right|^{2} d \mu_{\alpha, d}(x)\right) \frac{d a}{a} \\
\left.=\int_{\mathbb{R}_{+}^{d+1}}\left|\mathcal{F}_{W}^{\alpha, d}(f)(x)\right|^{2}\left(\frac{1}{C_{g}} \int_{0}^{\infty}\left|\mathcal{F}_{W}^{\alpha, d}(g)(a x)\right|^{2} \frac{d a}{a}\right) d \mu_{\alpha, d}(x)\right) .
\end{gathered}
$$

On the other hand, using relation (54), we have for almost all $x \in \mathbb{R}_{+}^{d+1}$

$$
\frac{1}{C_{g}} \int_{0}^{\infty}\left|\mathcal{F}_{W}^{\alpha, d} g(a x)\right|^{2} \frac{d a}{a}=1 .
$$

Then using the relation (18), we obtain

$$
\frac{1}{C_{g}} \int_{0}^{\infty} \int_{\mathbb{R}_{+}^{d+1}}\left|S_{g}^{W}(f)(a, x)\right|^{2} d \mu_{\alpha, d}(x) \frac{d a}{a}=\int_{\mathbb{R}_{+}^{d+1}}|f(x)|^{2} d \mu_{\alpha, d}(x) .
$$

The following theorem gives an inversion formula for the transform $S_{g}^{W}$. 
Theorem 6. Let $g$ be a Weinstein Wavelet on $\mathbb{R}_{+}^{d+1}$ in $L_{\alpha}^{2}\left(\mathbb{R}_{+}^{d+1}\right)$. For all $f$ in $L_{\alpha}^{1}\left(\mathbb{R}_{+}^{d+1}\right)$, respectively $L_{\alpha}^{2}\left(\mathbb{R}_{+}^{d+1}\right)$, such that $\mathcal{F}_{W}^{\alpha, d}(f)$ belongs to $L_{\alpha}^{1}\left(\mathbb{R}_{+}^{d+1}\right)$, respectively $L_{\alpha}^{1}\left(\mathbb{R}_{+}^{d+1}\right) \bigcap L_{\alpha}^{2}\left(\mathbb{R}_{+}^{d+1}\right)$, we have

$$
f(x)=\frac{1}{C_{g}} \int_{0}^{\infty}\left(\int_{\mathbb{R}_{+}^{d+1}} S_{g}^{W}(f)(a, y) g_{a, x}(y) d \mu_{\alpha, d}(x)\right) \frac{d a}{a}, \text { a.e, } \quad x \in \mathbb{R}_{+}^{d+1}
$$

The inner integral and the outer integral are absolutely convergent, but possibly not the double integral.

Proof. Using analogous proof as for Theorem 6. III. 3 of [15] page 99, we obtain the relation $(65)$

\section{Inversion formulas for the Weinstein intertwining op- erator and its dual using Weinstein wavelets}

In this section, we establish relations between the Weinstein continuous wavelet transform $S_{g}^{W}$ on $\mathbb{R}_{+}^{d+1}$ and the classical continuous wavelet transform $S_{g}$ on $\mathbb{R}_{+}^{d+1}$. Using the inversion formulas for the transforms $S_{g}^{W}$ and $S_{g}$, we deduce relations which give the inverse operators of the Weinstein intertwining operator $\mathcal{R}_{W}^{\alpha, d}$ and its dual ${ }^{t} \mathcal{R}_{W}^{\alpha, d}$.

Theorem 7. i) Let $g$ be a Weinstein Wavelet on $\mathbb{R}_{+}^{d+1}$ in $\mathcal{D}\left(\mathbb{R}^{d+1}\right)$, respectively $\mathcal{S}\left(\mathbb{R}^{d+1}\right)$. Then for all $f$ in the same space as $g$, we have for all $x \in \mathbb{R}_{+}^{d+1}$

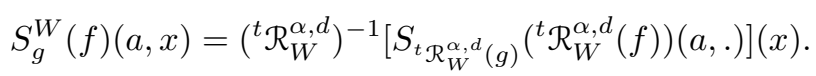

ii) Let $g$ be a Weinstein Wavelet on $\mathbb{R}_{+}^{d+1}$ in $\mathcal{S}_{0}^{0}\left(\mathbb{R}^{d+1}\right)$. Then for all $f$ in $\mathcal{S}_{0}\left(\mathbb{R}^{d+1}\right)$, we have

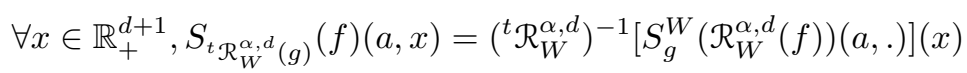

Proof. We deduce the relations (66) and (67) from the relations (51), (62) and properties of the Weinstein convolution product.

Theorem 8. Let $g$ be a Weinstein Wavelet on $\mathbb{R}_{+}^{d+1}$ in $\mathcal{S}_{0}^{0}\left(\mathbb{R}^{d+1}\right)$. Then i) For all $f$ in $\mathcal{S}_{0}^{0}\left(\mathbb{R}^{d+1}\right)$, we have for all $x \in \mathbb{R}_{+}^{d+1}$

$$
S_{g}^{W}(f)(a, x)=a^{-2 \alpha-1} \mathcal{R}_{W}^{\alpha, d}\left[S_{\mathcal{K}\left({ }^{t} \mathcal{R}_{W}^{\alpha, d}(g)\right)}\left({ }^{t} \mathcal{R}_{W}^{\alpha, d}(f)\right)(a, .)\right](x)
$$


ii)For all $f$ in $\mathcal{S}_{0}\left(\mathbb{R}^{d+1}\right)$, we have for all $x \in \mathbb{R}_{+}^{d+1}$

$$
S_{t \mathcal{R}_{W}^{\alpha, d}(g)}(f)(a, x)=a^{-2 \alpha-1}\left({ }^{t} \mathcal{R}_{W}^{\alpha, d}\right)\left[S_{\mathcal{K}_{\mathcal{W}}(g)}^{W}\left(\mathcal{R}_{W}^{\alpha, d}(f)\right)(a, .)\right](x)
$$

Proof. We obtain these relations from Theorem 7, Theorem 4 and the fact that

$$
\mathcal{K}\left[\left({ }^{t} \mathcal{R}_{W}^{\alpha, d}(g)\right)_{a}^{0}\right]=a^{-2 \alpha-1}\left[\mathcal{K}^{t} \mathcal{R}_{W}^{\alpha, d}(g)\right]_{a}^{0}
$$

and

$$
\mathcal{K}_{W}\left(g_{a}\right)=a^{-2 \alpha-1}\left(\mathcal{K}_{W}(g)\right)_{a} .
$$

Theorem 9. Let $g$ be a Weinstein Wavelet on $\mathbb{R}_{+}^{d+1}$ in $\mathcal{S}_{0}^{0}\left(\mathbb{R}^{d+1}\right)$. Then: i) for all $f$ in $\mathcal{S}_{0}^{0}\left(\mathbb{R}^{d+1}\right)$, we have for all $x \in \mathbb{R}_{+}^{d+1}$

$$
\begin{gathered}
\left({ }^{t} \mathcal{R}_{W}^{\alpha, d}\right)^{-1}(f)(x) \\
=\frac{1}{C_{g}} \int_{0}^{\infty}\left(\int_{\mathbb{R}_{+}^{d+1}} \mathcal{R}_{W}^{\alpha, d}\left[S_{\mathcal{K}\left({ }^{t} \mathcal{R}_{W}^{\alpha, d}(g)\right)}(f)(a, .)\right](y) g_{a, x}(y) d \mu_{\alpha, d}(y)\right) \frac{d a}{a^{2 \alpha+2}} ;
\end{gathered}
$$

ii) for all $f$ in $\mathcal{S}_{0}\left(\mathbb{R}^{d+1}\right)$, we have for all $x \in \mathbb{R}_{+}^{d+1}$

$$
\begin{gathered}
\left(\mathcal{R}_{W}^{\alpha, d}\right)^{-1}(f)(x) \\
=\frac{1}{C_{t_{\mathcal{R}_{W}}^{\alpha, d}(g)}^{0}} \int_{0}^{\infty}\left(\int_{\mathbb{R}_{+}^{d+1}} t \mathcal{R}_{W}^{\alpha, d}\left[S_{\mathcal{K}_{W}(g)}^{W}(f)(a, .)\right](y)^{t} \mathcal{R}_{W}^{\alpha, d}(g)_{a, x}(y) d \mu_{\alpha, d}(y)\right) \frac{d a}{a^{2 \alpha+2}} .
\end{gathered}
$$

Proof. We deduce the relations (69) and (69) from Theorem 8, Theorem 3 and the relation $(53)$

\section{Acknowledgment}

This research project ( grant number 6032/1435 ) supported by the Scientific Research Deanship at Taibah University. Al-Madinah Al-Munawarah, Kingdom of Saudi Arabia.

\section{References}

[1] I. A. Aliev and B. Rubin, Parabolic potentials and wavelet transform with the generalized translation, Studia Math. 145 (2001) Nr1, p. 1-16.

[2] H. Ben Mohamed, N. Bettaibi and S. H. Jah, Sobolev type spaces associated with the Weinstein operator, Int. Journal of Math. Analysis, Vol. 5, Nr. 28, (2011), p. 1353-1373. 
[3] H. Ben Mohamed, B. Ghribi, Weinstein-Sobolev spaces of exponential type and applications. To appear in Acta Mathematica Sinica, English Series (2012)p. 1-18.

[4] Z. Ben Nahia, Fonctions harmoniques et proprietés de la moyenne associées à l'opérateur de Weinstein, Thèse $3^{\grave{e ̀ m e}}$ cycle Maths. (1995) Department of Mathematics Faculty of Sciences of Tunis. Tunisia.

[5] Z. Ben Nahia and N. Ben Salem, Spherical harmonics and applications associated with the Weinstein operator, "Proceedings" de la Conférence Internationale de Théorie de Potentiel, I. C. P. T. 94, tenue à Kouty ( en République Tchèque ) du 13-20 Août 1994.

[6] Z. Ben Nahia and N. Ben Salem, On a mean value property associated with the Weinstein operator, "Proceedings" de la Conférence Internationale de Théorie de Potentiel, I. C. P. T. 94, tenue à Kouty ( en République Tchèque ) du 13-20 Août 1994.

[7] M. Brelot, Equation de Weinstein et potentiels de Marcel Riesz, Lecture Notes in Mathematics 681, Séminaire de Théorie de Potentiel Paris, No 3, 1978, 18-38.

[8] C. Chettaoui and K. Trimeche, Bochner-Hecke theorems for the Weinstein transform and application, fract. cal. Appl. Anal. 13(3) (2010) 261-280.

[9] I. A. Kipriyanov, Singular Elliptic Boundary Value Problems, Nauka, Fizmatlit, 1997 (in Russian).

[10] T. H. Koornwinder, The continuous wavelet transform, in wavelets. An elementury treatment of theory and applications, T. H. Koornwinder, ed., World Scientific, (1993).

[11] J. Löfström and J. Peetre, Approximation theorems connected with generalized translations Math. Ann. Nr 181 (1969), p. 255-268.

[12] H. Mejjaoli, Heat Equations Associated with Weinstein Operator and Applications, Journal of Function Spaces and Applications, Volume 2013, Article ID 723976, 13 pages.

[13] H. Mejjaoli and A. O. A. Salem, Weinstein Gabor Transform and Applications, Adv. in Pure Math. 2 (2012) 203-210.

[14] K. Stempak, La théorie de Littlewood-Paley pour la transformation de Fourier-Bessel, C. R. Acad. Sci. Paris Sér. I303 (1986), p. 15-18. 
INVERSION OF THE WEINSTEIN INTERTWINING OPERATOR AND ITS

[15] K. Trimèche, Generalized Wavelet and Hypergroups, Gordon and Breach, New York, 1997.

[16] K. Trimèche, Generalized harmonic analysis and wavelet packets, Gordon and Breach Science Publishers, 2001.

Abdessalem Gasmi,

Department of Mathematics,

Faculty of Sciences,

Taibah University, Medina, Saudi Arabia.

Email: aguesmi@taibahu.edu.sa

Hassen Ben Mohamed,

Department of Mathematics,

Faculty of Sciences of Gabes,

Gabes University, Gabes, Tunisia.

Email: hassenbenmohamed@yahoo.fr

Néji Bettaibi,

Department of Mathematics,

Faculty of Sciences,

Qassim University, Buraydah, Saudi Arabia.

Email: neji.bettaibi@ipein.rnu.tn 